\title{
Progress in multi-channel all-optical regeneration based on fiber technology
}

\author{
Francesca Parmigiani, Lionel Provost, Periklis Petropoulos, David J. Richardson, Wolfgang Freude, \\ Senior Member, IEEE, Juerg Leuthold, Senior Member, IEEE, Andrew D. Ellis, and Ioannis Tomkos, \\ Senior Member, IEEE
}

(Invited Paper)

\begin{abstract}
Multi-wavelength all-optical regeneration has the potential to substantially increase both the capacity and scalability of future optical networks. In this paper we review recent promising developments in this area. First we recall the basic principles of multi-channel regeneration of high bit rate signals in optical communication systems before discussing the current technological approaches. We then describe in detail two fiber-based multi-channel $2 \mathrm{R}$ regeneration techniques for RZOOK based on (a) dispersion managed systems and (b) direction and polarization multiplexing. We present results illustrating the levels of performance so far achieved and discuss various practical issues and prospects for further performance enhancement.
\end{abstract}

Index Terms-Nonlinear optical, devices; Kerr effect; signal regeneration; nonlinear optical signal processing; ultrafast processes in fibers.

\section{INTRODUCTION}

IGNAL regeneration is a key functionality and can be used $N_{\text {to increase the reach of transmission systems and to }}$ provide increased flexibility and scalability in network design. Current commercial regenerators are based exclusively on optoelectronic regeneration techniques, whereby the optical signal is received, converted into the electrical domain where it is regenerated, before conversion back to the optical domain if further transmission is required. This is a mature and powerful solution. However, optical-to-electrical-to-optical (O-E-O) approaches face significant issues in terms of

Manuscript received November 14, 2010. This work was supported in part by the European projects TRIUMPH (grant IST-027638 STP) and NoE BONE (216863), and the Science Foundation Ireland (grant 06/IN/I969). Dr F. Parmigiani gratefully acknowledges the support from the Royal Academy of Engineering/EPSRC through a University research Fellowship.

F. Parmigiani, P. Petropoulos, D. J. Richardson are with the Optoelectronics Research Centre, University of Southampton, Southampton SO17 1BJ, U.K., (phone: +44 238059 3141; email: frp@orc.soton.ac.uk).

L. Provost is with Draka Communications, France.

W. Freude and J. Leuthold are with the Institute of Photonics and Quantum Electronics, University of Karlsruhe, 76131 Karlsruhe, Germany.

A. D. Ellis is with the Tyndall National Institute and Department of Physics, University College Cork, Ireland.

I. Tomkos is with Athens Information Technology Centre, Athens, Greece. scalability due to electronic speed limitations, which will limit line rates and power requirements if the regeneration of a large number of channels is required. The cost of implementation is also an issue as higher and higher speed electronics is needed.

Several all-optical 2R (re-amplification, re-shaping) and 3R (re-amplification, re-shaping, re-timing) techniques have also been demonstrated and reported in the literature over the years [1-4] - many providing excellent regenerative performance. The vast majority of these techniques support only single optical channel operation [2-8], limited in terms of multichannel operation by the strong inter-channel crosstalk effects that arise when the same nonlinear medium is simultaneously used for the regeneration of all channels. However, in order for all optical regenerators to offer a commercially interesting alternative to their optoelectronic counterparts, extension to multi-channel operation is likely to be essential [9]. Consequently, all-optical regeneration capable of simultaneously processing multiple wavelength-divisionmultiplexed (WDM) channels at any bit rate has attracted significant attention over the last few years as it would represent considerable added value.

To date, numerous nonlinear gates have been demonstrated for regeneration, mainly based on semiconductor optical amplifiers (SOAs) [10-13], optical fibers [2-8], saturable absorbers [14-15], synchronous modulation associated with narrow band filtering [16-17] and electro absorption modulators (EAMs) [18-19]. However, only a few of them are truly appropriate candidates for high bit rates and high capacity WDM systems. Quantum dot SOAs (QD-SOAs) could potentially provide multi-wavelength operation at high bit rates [11-13], thanks to their saturated gain response time (of the order of $100 \mathrm{fs}$ to $1 \mathrm{ps}$ ), leading to negligible patterning effects, and spatial isolation of dots, leading to spectrally localized effects and, thus, to crosstalk suppression between WDM channels under gain saturation conditions. However, very little experimental work has been published to date [20] demonstrating their multi-wavelength operation in support of the encouraging numerical predictions [13].

Fiber-based nonlinear gates are bulkier compared to their competing technologies, but are very attractive due to their potential to operate at high bit rates due to the fs response time 
of the Kerr effect and the great design flexibility for tailoring the optical fiber properties. This paper reviews some of the techniques that allow multi-wavelength regeneration to be achieved in fiber-based systems, and outlines some of the results achieved to date. The paper is organized as follows: in section II we discuss the recent developments in the area of multi-wavelength all-optical regeneration briefly summarizing work from various research groups across the world. We mainly focus on the various techniques and corresponding operational principles used to extend from single to multichannel operation. In sections III and IV we present some of the results on multi-wavelength all-optical regeneration suitable for high bit rates, applicable to Return-to-Zero (RZ) On-Off Keying (OOK) modulation formats, achieved within the European research project TRIUMPH [21-22]. In this project we mainly focused on two types of all-optical $2 \mathrm{R}$ regenerator. Both of them are based on the generation of selfphase modulation (SPM)-induced spectral broadening in fibers followed by optical filtering at offset wavelengths. The two schemes differ in their approach to mitigation of the interchannel nonlinearities and are based on either uni- or bidirectional architectures. In more detail, section III presents the key operational principles of the uni-directional configuration and the corresponding experimental demonstrations for four $10 \%$ duty-cycle RZ 10Gbit/s channels and up to three $33 \% \mathrm{RZ} 43 \mathrm{Gbit} / \mathrm{s}$ channels, in both cases using a channel-spacing of $600 \mathrm{GHz}$. Section IV reports the corresponding results for the bi-directional configuration together with a polarization multiplexing scheme enabling the simultaneous regeneration of up to two $130 \mathrm{Gbit} / \mathrm{s}$ channels and up to four $10 \mathrm{Gbit} / \mathrm{s}$ channels. Finally, in section V, we draw our main conclusions and discuss future trends on multiwavelength regeneration.

\section{REVIEW OF MULTI-WAVELENGTH FIBER BASED ALL- OPTICAL REGENERATORS}

In general, single channel all-optical regenerators can be achieved through in-line synchronous modulation and narrowband filtering [16-17], or they can be based on exploiting nonlinear optical gates [2-5] which might, for example, be based on an optical fiber designed and configured to exploit ultrafast nonlinear effects such as Self-Phase modulation, Cross-Phase modulation (XPM), Four-Wave mixing (FWM), or Stimulated Raman Scattering.

The extension to simultaneous multi-channel operation is generally limited by the presence of deleterious nonlinear interactions among the various channels, the so-called interchannel crosstalk, which directly competes with the nonlinear effect used for the regeneration process itself. In fibers, such crosstalk typically results from XPM and FWM interactions, which manifest themselves in terms of strong spectral distortions (often asymmetric) and spectrally dependent power transfer due to partial collisions of co-propagating pulses during nonlinear propagation. Fixed relationships between all the WDM channels in terms of bit sequences, channel powers (a) Space division multiplexing

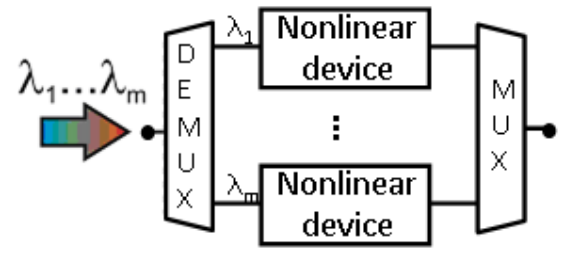

(b) Time interleaving

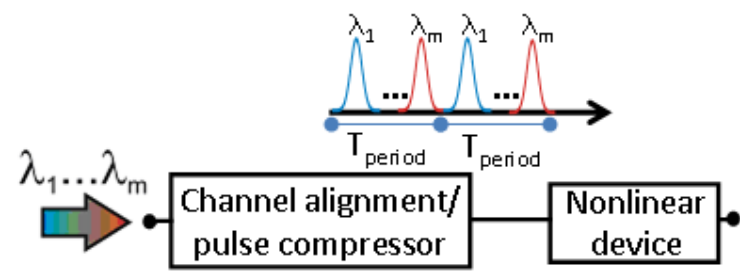

(c) Dispersive walk-through
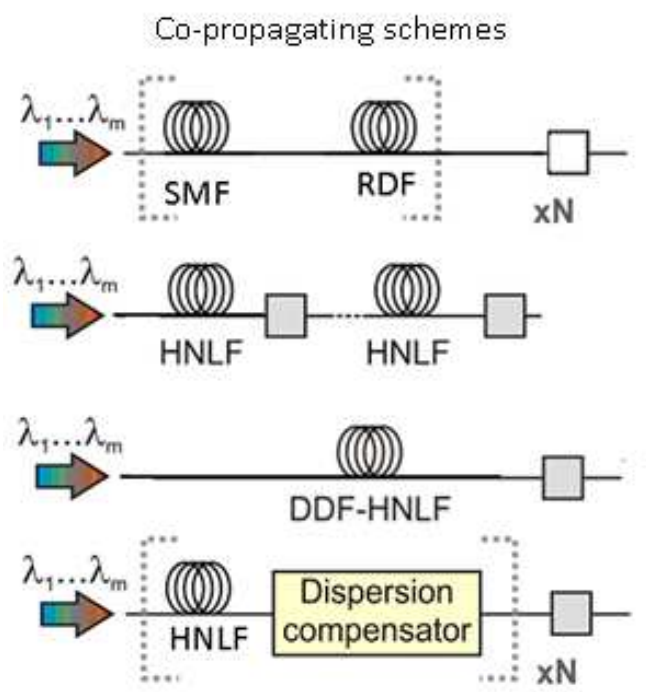

Counter-propagating scheme

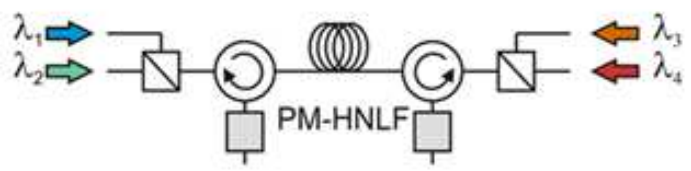

Fig. 1. Schematic of multi-channel $2 \mathrm{R}$ regenerators based on (a) spacedivision demultiplexing, (b) time interleaving and (c) dispersive walkthrough in co-propagating geometries, exploiting spectrally filtered optical solitons (i), quasi-continuous filtering (ii), dispersion decreasing fibers (iii) and dispersion managed fibers (iv), or in counter-propagating geometry (v). Empty squares represent carrier-centered filters, while gray squares represent carrier-offset filters. RDF: Reverse Dispersion Fiber.

and timings would be required to avoid (or at least compensate) for such crosstalk, which is obviously not the case in general for real operational systems. However, mitigation of such inter-channel nonlinearities can be achieved by ensuring that the pulses within adjacent channels 
completely walk-through each other, so that the corresponding crosstalk can be averaged out. Then, the XPM-induced nonlinear phase shift imparted across the full envelope of a pulse can be considered to be either null or at least constant. Moreover, the negligible or short effective interaction length restricts the impact of any FWM phase matching conditions. So far, attempts to regenerate more than one WDM channel have employed various approaches and technologies (Fig.1).

In the first instance, space-division multiplexing is used to allow each of the channels to be processed in separate but identical regenerators, see Fig.1 (a) [14-15, 23-26]. After regeneration, the channels are then recombined together through a wavelength multiplexer into a single fiber for further transmission. This implementation has been demonstrated exploiting several nonlinear systems, including Mach-Zehnder modulators [23], saturable absorbers [14-15] and fiber-based nonlinear optical loop mirrors [24-26]. In more detail, O. Leclerc et al. reported in [23] the very first demonstration in a transmission loop of simultaneous optical regeneration of four RZ OOK $200 \mathrm{GHz}$ spaced 40Gbit/s channels, allocating a soliton compression regenerator [26] to each channel followed by a common InP Mach-Zehnder modulator to achieve full 3R regeneration. Using the same approach, $\mathrm{K}$. Cvecek et al. have more recently demonstrated a phase preserving amplitude regenerator for two 10Gbit/s RZ differential phase shift keying (DPSK) signals using a nonlinear amplifying loop mirror with a penalty less than $0.2 \mathrm{~dB}$ in the receiver input power between single- and multi-channel operation [26].

Such a per-channel scheme clearly avoids crosstalk- induced wave distortion inside the nonlinear device; however, the complexity and cost of the scheme increases linearly as the number of channels to be simultaneously regenerated increases.

In a second approach, inter-channel crosstalk is avoided by time interleaving the input signals [28-32]. In these schemes, the various WDM channels are properly spaced in time before entering the regenerator, see Fig.1 (b). This approach has been investigated in both fiber [28-31] and SOA technologies [32]. The WDM channels are then processed at once in a serial fashion in a single nonlinear medium, guaranteeing a reduction of cost as the number of channels increases. However, the WDM channels need to be synchronous and with a relatively low duty cycle, since the maximum pulse width that a channel can occupy is determined by the number of WDM channels simultaneously processed by the regenerator. The WDM synchronicity can be achieved by either using appropriate delay lines located within a demultiplexer/multiplexer apparatus [30-32] or making the WDM channels properly time-aligned at the regenerator input using specific wavelength-mapping rules [28-29]. To achieve the correct duty cycle, a pulse compression stage is likely to be needed prior to the regenerator (see Fig.1 (b)), making, however, the use of the bandwidth of the transmission line relatively inefficient and the whole arrangement quite complicated as the number of WDM channels increases. For example in [31], a pulse duty ratio of $\sim 6 \%$ was used for simultaneous regeneration of 2 OOK $10 \mathrm{Gbit} / \mathrm{s}$ channels, spaced by $1.2 \mathrm{~nm}$. However, according to their preliminary numerical and experimental studies, duty ratios up to $20 \%$ would be acceptable for such an approach [31].

Finally, in a third approach, which has been developed for WDM OOK signals, carefully engineered dispersive walkthrough maps in fibers are used to avoid the inter-channel crosstalk [30, 33-48]. In these schemes a high local average dispersion value ensures that the nonlinear inter-channel effects are significantly weaker due to the fast walk-off time among the various WDM channels, while a low overall average dispersion value ensures that the nonlinear effect used for the regeneration process itself (SPM) is sufficiently retained, Fig.1 (c). Also in this approach, the WDM channels can be processed simultaneously in a single medium, guaranteeing a reduction of cost as the number of channels increases. However, while the previous two approaches could potentially use any kind of nonlinear device and nonlinearity, this third approach may only be based on SPM in fibers and is thus only applicable to OOK signals. It is, though, worth pointing out that, to date, SPM-based regenerators are the most promising ones due to their simple implementation and excellent performance and thus the easiest to be adapted for multi-wavelength operation if OOK signals are used.

These types of SPM-based regenerators differ in their architectures, according to whether the various WDM channels all propagate in the same direction [30, 33-41] or some in opposite directions [42-48] within the nonlinear fiber, see Fig. 1 (c.i-v). This can either be through a carefully tailored highly nonlinear fiber (HNLF) [41-48], a dispersion decreasing fiber (DDF) [33] or chromatic dispersion-managed fiber assemblies [30, 34-40]. Decision-threshold based filtering at the regenerator output may then be performed in a variety of different ways, namely the filter may be centered at the same wavelength as the original signal (usually referred to as a spectrally filtered optical soliton regenerator) [40], see Fig. 1 (c.i), centered at an offset wavelength from the original signal (usually referred to as Mamyshev regenerator in the case of single channel operation) [30, 33-39, 42-49], see Fig. 1 (c. iii$\mathrm{v}$ ), or progressively offset relative to the signal wavelength in successive regeneration steps [41], see Fig.1 (c.ii).

The all-optical multichannel limiters that use spectrally filtered optical solitons rely on progressive pulse reshaping of the incoming signal during propagation in an anomalous HNLF at a precise input power (see Fig.1 (c.i)). Narrow-band filtering around the carrier frequency allows for the removal of any spectral content generated from intensity fluctuations present at the input. While it guarantees a relatively efficient spectral bandwidth usage for each WDM channel, the drawback of the scheme is that it cannot remove in-band noise.

The extension of the Mamyshev regenerator [8] to multiwavelength operation, on the other hand, relies on the dependence of SPM induced spectral broadening on the gradient of the pulse intensity profile in a HNLF, followed by 
an offset filter to carve the broadened spectrum (see Fig.1 (c.iiv)). This scheme can, thus, provide pulse reshaping on the marks and noise removal from the spaces, thereby better discriminating between "ones" and "zeros". However, a relatively large spectral spacing between adjacent WDM channels has to be guaranteed to allow optimum regenerative capabilities.

In the following two sections we report the studies of the two main routes we have recently investigated, applicable to RZ OOK modulation formats, which are based on this third approach, i.e. exploiting co- and counter- propagation geometries in dispersive walk-through schemes.

\section{UNI-DIRECTIONAL SCHEME FOR MULTI-WAVELENGTH REGENERATION}

\section{A. Operation principle}

Uni-directional geometries are very attractive since the incoming signals can in principle be processed in-line without the need for any channel demultiplexing, and can potentially allow scalability of the scheme to a large number of WDM channels. Indeed, Patki et al. have recently demonstrated $12 \times 10 \mathrm{Gbit} / \mathrm{s}$ all-optical $2 \mathrm{R}$ regeneration with $200 \mathrm{GHz}$ spacing using a group-delay management scheme (see Fig. 2 (b)) in such a geometry [34]. Within the TRIUMPH project, two unidirectional approaches were considered based on either soliton-like pulse compression in anomalous dispersion, followed by carrier-centered filtering [40] or spectral broadening in normal dispersion followed by carrier-offset filtering [38]. The two corresponding experimental implementations are reported in Fig.2 (a) and (b), respectively. In both cases, nonlinear inter-channel interference was avoided by employing multiple fiber sections with alternating signs of dispersion. This allowed the dispersion to be kept high locally, thereby introducing rapid walk-off between adjacent channels, while the pulse integrity and efficient spectral broadening was maintained by virtue of a low path average chromatic dispersion over the regenerator length, as discussed in the previous section for the third approach.

Although both types of regenerator show good regeneration performance, the extension of the Mamyshev based regenerator (offset filtering case) provides better ability to stabilize the signal amplitude, albeit at the cost of a higher signal power launched into the HNLF [50]. Furthermore, the spectrally filtered soliton regenerator cannot suppress the noise in the spaces [36]. For these reasons only the type of regenerator sketched in Fig. 2 (b) was further investigated and in a new regime (anomalous net dispersion of the regenerator), which was found to improve its performance [37].

The original design of this regenerator comprised sections of nonlinear fiber (e.g. dispersion compensating fiber (DCF)) followed by a periodic group delay device (PGDD) with a saw-tooth-like profile, which acted as a channelized dispersion compensator [34-35, 37-38]. This configuration allowed the channels to uni-directionally walk through each other, reducing any bit-pattern dependence of the nonlinear crosstalk.
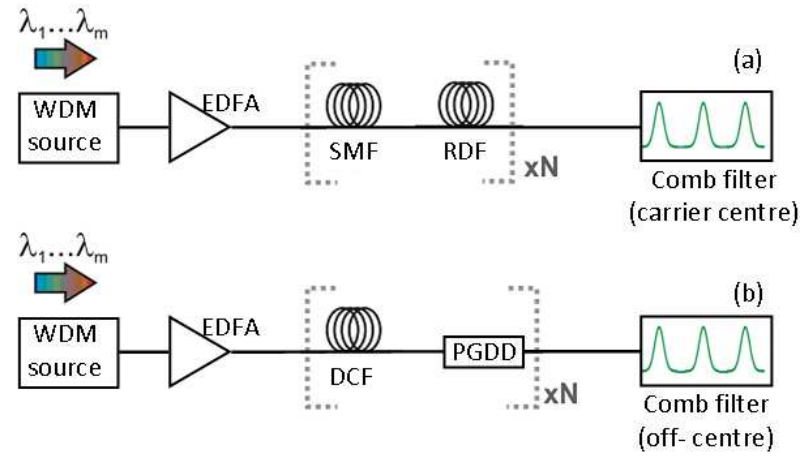

Fig. 2. Configurations of the co-propagating multi-wavelength regenerators after Ref. [40] and [38], respectively.

\section{(a)}

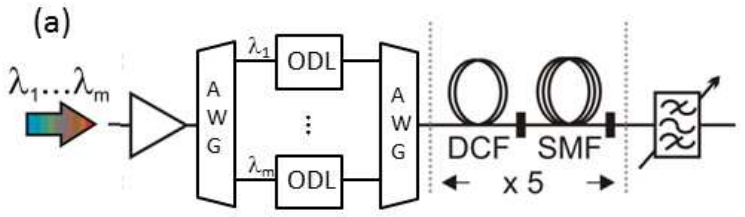

(b)

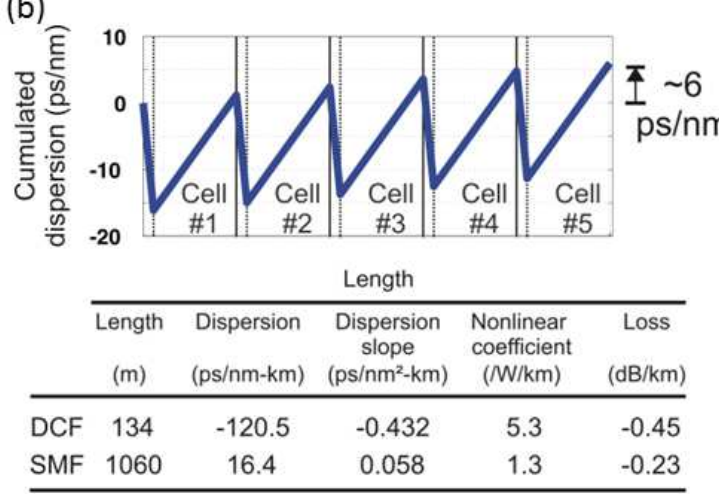

Fig. 3. (a) Experimental set-up of the dispersion managed regenerator for simultaneous processing of $4 \times 10 \mathrm{Gbit} / \mathrm{s}$ and $3 \times 40 \mathrm{Gbit} / \mathrm{s}$ channels. (b) Fibers parameters and dispersion map of the device (at 1550nm).

It was later shown that the more exotic PGDDs could be replaced by conventional fiber dispersion compensation modules, such as lengths of standard single mode fiber (SMF), which have a monotonic rather than periodic group delay function, implying that the pulses of the adjacent channels walk back and forth along the fiber elements and interact repeatedly with a prescribed set of neighboring pulses [37, 30]. Consequently, the mitigation performance shows a slightly higher dependence on the relative time delay between incoming channels as compared to the former solution, as the group delay difference, GDD, (given as $\mathrm{GDD}=\left|\mathrm{D}_{\mathrm{i}}\right| \times \mathrm{L}_{\mathrm{i}} \times \Delta \lambda$, where $\mathrm{D}_{\mathrm{i}}$ is the fiber dispersion, $\mathrm{L}_{\mathrm{i}}$ is the fiber length and $\Delta \lambda$ is the channel spacing) between adjacent channels is equal to a few bit slots only. Good performance is still achievable as it will be shown in the following subsection. However, higher interchannel spacing was adopted as compared to Ref. [37].

\section{B. Experimental validations}

Fig.3 (a) depicts the core of the all-fiberized dispersion 

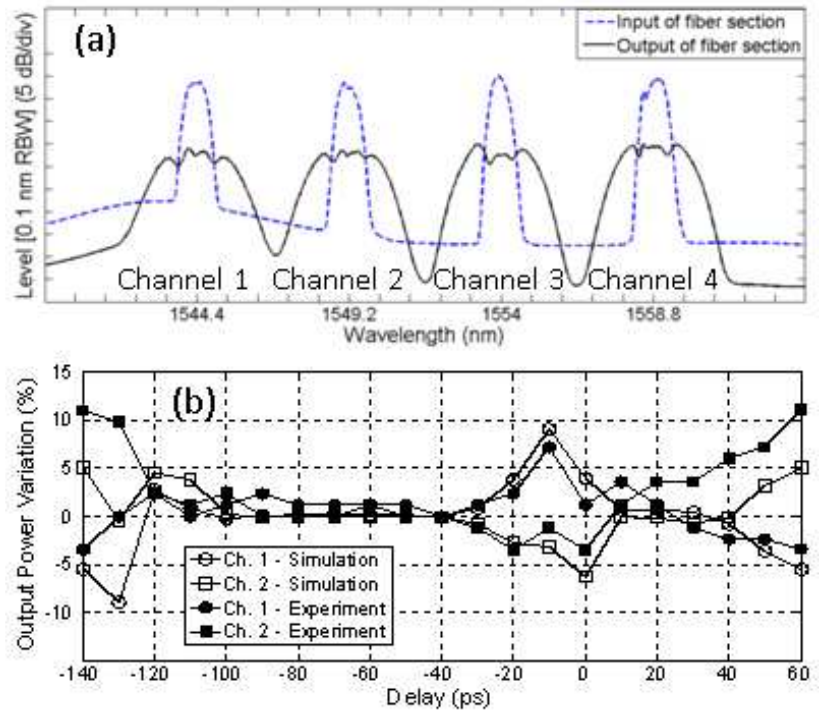

Fig. 4. (a) Spectrum of the WDM signal at the input/output for the $4 \times 10 \mathrm{Gbit} / \mathrm{s}$ case. (b) Output power variation of channel 1 and 2 at the bit rate of $5 \mathrm{GHz}$, compared to the single-channel case, for different values of time delay between the two channels. Positive delay values indicate precedence of channel 1 .

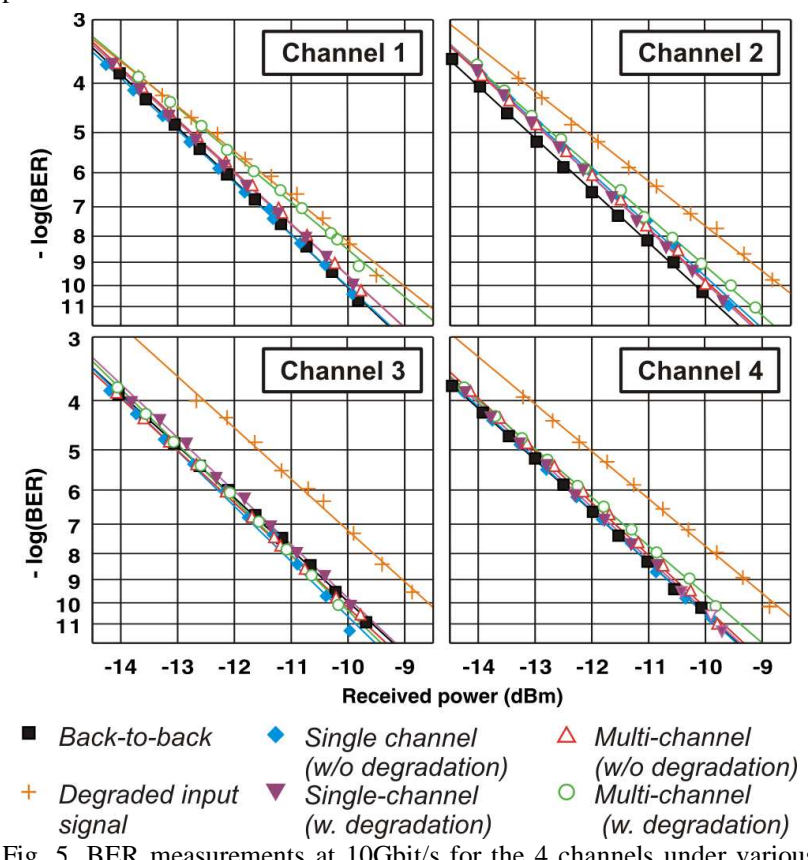

Fig. 5. BER measurements at $10 \mathrm{Gbit} / \mathrm{s}$ for the 4 channels under various signal degradation and operating conditions.

managed regenerator which we experimentally implemented and used to validate the scheme discussed in the previous subsection. Five alternating DCF-SMF sections with a total length of $\sim 6 \mathrm{~km}$, overall loss of $\sim 9 \mathrm{~dB}$ (mainly due to the splices between DCF and SMF, which were of the order of $1 \mathrm{~dB} /$ splice) and a residual chromatic dispersion of a few $\mathrm{ps} / \mathrm{nm}$ were used as shown in Fig.3 (b). This dispersion map provided a maximum excursion of $\sim 75$ ps GDD for a $600 \mathrm{GHz}$ channel separation within each cell. The fiber assembly was then followed by a tunable optical filter to perform decision thresholding at an offset wavelength [30, 39]. Using this dispersion map, we successfully demonstrated up to $4 \times 10 \mathrm{~Gb} / \mathrm{s}$ (a)
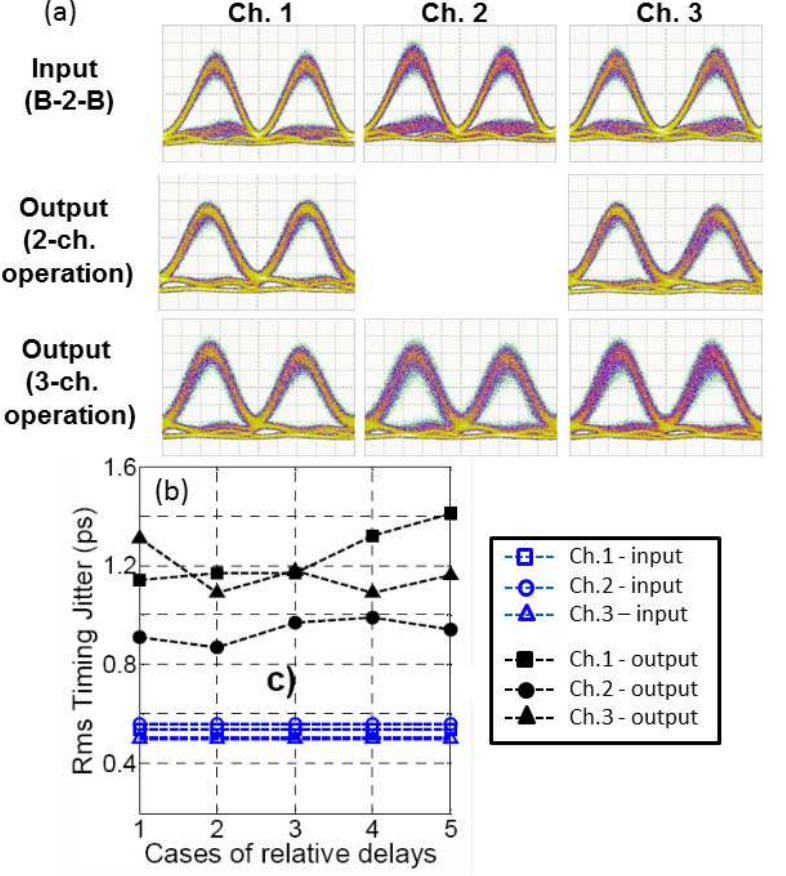

Fig. 6. (a) Eye diagrams for the $3 \times 43 \mathrm{Gbit} / \mathrm{s}$ case. Top row: degraded input channels. Middle row: regenerator output for dual-channel operation (1200 GHz spectral separation). Bottom row: regenerator output for threechannel operation $(600 \mathrm{GHz}$ spectral separation). (b) Timing jitter measurements in three-channel operation at $43 \mathrm{Gbit} / \mathrm{s}$ with $600 \mathrm{GHz}$ spacing and degraded input signal when 5 randomly chosen combinations of relative time delays were considered.

[30] and $3 \times 43 \mathrm{~Gb} / \mathrm{s}$ [39] regeneration. The WDM channel spacing in both cases was $600 \mathrm{GHz}$.

Firstly, four WDM channels ( $~ 8 \mathrm{ps}$ long pulses, $\sim 8 \%$ duty cycle) were independently amplitude modulated at $10 \mathrm{Gbit} / \mathrm{s}$, properly power equalized and time delayed, before being multiplexed together to enter the regenerator, shown in Fig. 3 (a). The total average power at the input of the regenerator was about $27 \mathrm{dBm}$. Fig.4 (a) shows the corresponding spectrum at the input and output of the fiber assembly, indicating significant SPM-induced broadening.

A first investigation of the inter-channel nonlinear crosstalk was conducted by studying the impact of the initial time delay, $\tau$, between two adjacent channels (Channels 1 and 2 were chosen) at the device input as a function of their output powers. The output power variations relative to the singlechannel case for each channel are summarized in Fig. 4 (b), where a maximum variation of $11 \%$ is observed. The results were qualitatively confirmed by simulations, see Fig. 4 (b).

The actual regenerator performance was investigated by biterror-rate (BER) measurements (see Fig. 5), showing that the effects of the inter-channel nonlinearities were mitigated with negligible power penalty between the cases of multiple- and single- channel operation, in the presence or absence of any signal degradation.

Successful operation of the system was subsequently confirmed at $43 \mathrm{Gbit} / \mathrm{s}$ with $33 \%$ duty cycle pulses under single-, two-, and three- channel operation. The spectral 
separations for the two- and three- channel systems were 1200 $\mathrm{GHz}$ and $600 \mathrm{GHz}$ respectively. The dispersion map and experimental set-up were maintained the same as in Fig.3. The average power per channel at the input of the fiber assembly was adjusted at $25.5 \mathrm{dBm}$ in all cases.

Complete BER measurements were taken to evaluate the system performance, both for single- and multi-channel operation and in the presence or absence of artificially induced noise [39]. Two-channel operation allowed a power penalty improvement of $2.5 \mathrm{~dB}$ over a degraded input signal. Threechannel operation was still feasible albeit at the expense of slightly degraded performance due mainly to an increased XPM-induced timing jitter contribution which caused some BER floor in the measurements [39]. This effect is clearly visible from the corresponding eye-diagrams shown in Fig. 6 (a). A measurement of the timing jitter is reported in Fig.6 (b) taken from the corresponding eye-diagrams, when threechannel operation and a degraded input signal were considered.

\section{Bi-Directional, Polarization MultiPleXing SCHEME FOR MULTI-WAVELENGTH REGENERATION}

\section{A. Operation principle}

Similar to the scheme discussed in the previous section, the optical $2 \mathrm{R}$ regenerator based on the counter-propagating architecture relies on the scheme proposed by Mamyshev [49], where now the two incoming signals propagate in the same HNLF but in opposite directions, see Fig.1 (c.v). The great benefit of this bi-directional scheme is that the inclusion of the second channel does not compromise at all the performance of the regenerator, as compared to its operation with a single channel, preserving its general operating principles. In this way, the two-channel regenerator can be designed following the same rules as those applied for the single-channel operation [51].

Fig. 7 (a) schematically illustrates the implementation of the bi-directional architecture. As previously discussed, to alleviate the nonlinear crosstalk between adjacent channels, one has to minimize the interaction time between pulses by ensuring a complete and fast walk-through. The major benefit of this solution lies in the extremely high relative walk-off value, $\mathrm{T}_{\mathrm{W}}$, between the two counter-propagating channels, which is typically $\sim 2 / \operatorname{Vg}(\lambda)$, where $\operatorname{Vg}(\lambda)$ is the group velocity at wavelength $\lambda$. In case of silica-based fibers for example, $T_{W}$ is about $10 \mathrm{~ns} / \mathrm{m}$ and is almost independent of the fiber properties and the spectral allocation of the channels. The immediate result of a high $\mathrm{T}_{\mathrm{W}}$ value is that any individual data pulse interacts with a large number of counter-propagating data pulses of the second channel, experiencing the same net average interaction. The accumulated nonlinear phase $\phi_{\mathrm{NL}}$ induced by XPM manifests itself as a constant phase shift across the whole pulse (proportional to the ratio between the pulse peak power and $\mathrm{T}_{\mathrm{W}}$ ), as shown in Fig. 7 (b), generating no additional frequencies and, thus, reducing any induced distortion on the SPM-broadened spectrum. Similarly, because of the relatively high walk-off, there is no FWM phase- (a)

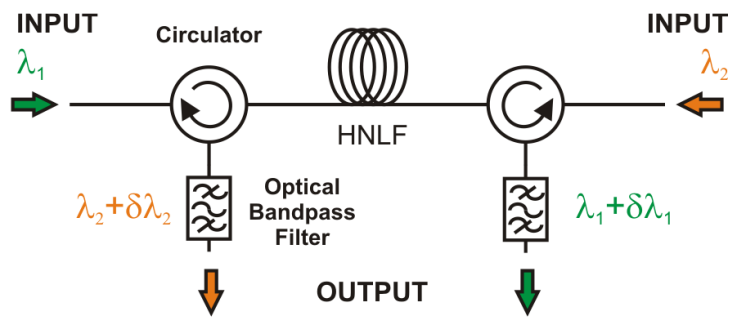

(b)
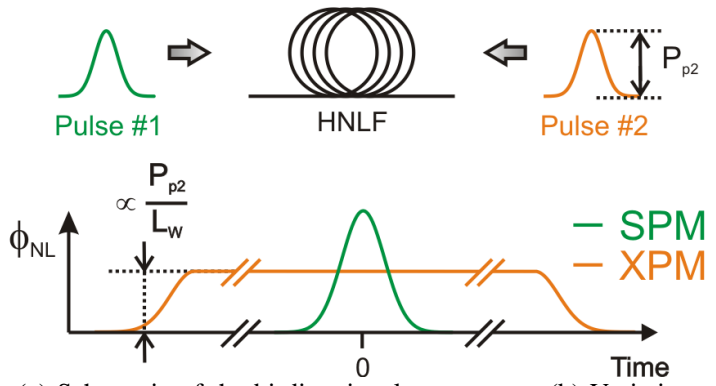

Fig. 7. (a) Schematic of the bi-directional regenerator. (b) Variation of the nonlinear phase self-induced on the first pulse by SPM (green curve) and XPM (orange curve) arising from the interaction with a second counterpropagating pulse. The NL phases are plotted across the temporal window of the first pulse.

(a) Ch. 1

$\begin{array}{llllllll}0 & 0 & 1 & 0 & 0 & 0 & \text { PM-HNLF } & \begin{array}{r}\text { Passban } \\ \text { Filter }\end{array}\end{array}$

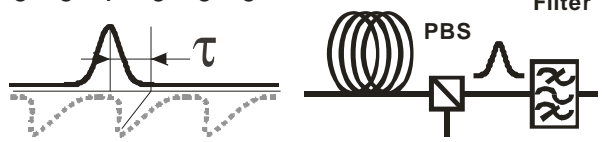

$\begin{array}{llllll}1 & 0 & 1 & 0 & 1 & 0\end{array}$

Ch. 2

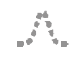

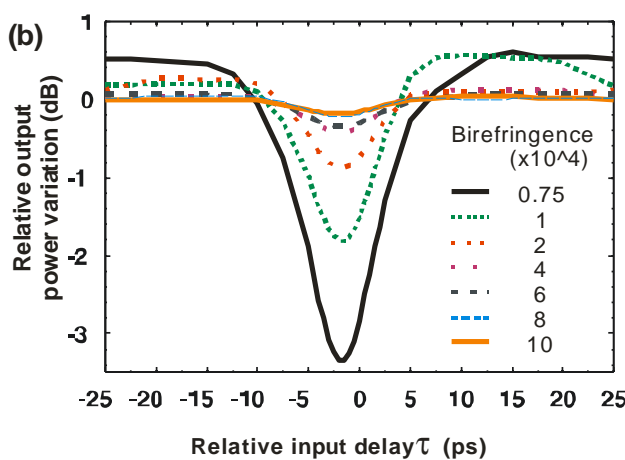

Fig. 8. (a) Operation principle. (b) Influence of birefringence on the TFs variation obtained at the flat top point of the TF as a function of the initial delay. The co-propagating pulses were $8 \mathrm{ps} 600 \mathrm{GHz}$ spaced $10 \mathrm{Gbit} / \mathrm{s}$ Gaussian of alternating marks and spaces, while the optical fiber parameters used correspond to those reported in the corresponding experimental section.

matching condition between the two counter-propagating pulses. Despite the reduction in the XPM and FWM crosstalk, the proposed bi-directional architecture introduces a new source of crosstalk resulting from any reflections of the counter-propagating signal as it propagates along the HNLF. Since these reflections co-propagate with the second signal, they may give rise to some performance degradation, and therefore their contribution was thoroughly investigated in [45].

These reflection contributions come from either local defects generating a strong signal reflection (e.g. reflections at connections and splices, or extraordinary defects in the fibers), or more predominantly from the backscattering experienced 

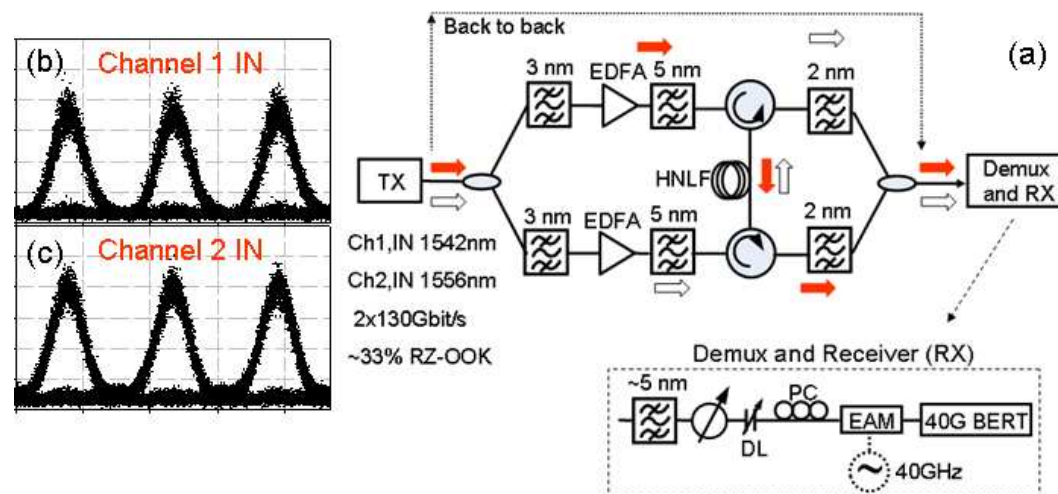

(a)

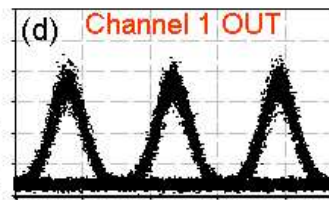

(e) Channel 2 OUT

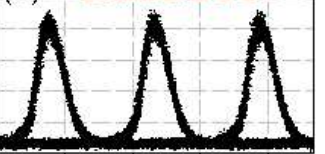

Fig. 9. a) $2 \mathrm{R}$ regenerator scheme. EDFA: erbium-doped fibre amplifier, DL: delay line, PC: polarisation controller, TX: transmitter. b-e) Eye diagrams of the two $130 \mathrm{Gbit} / \mathrm{s}$ channels at the input/output ports of the regenerator.
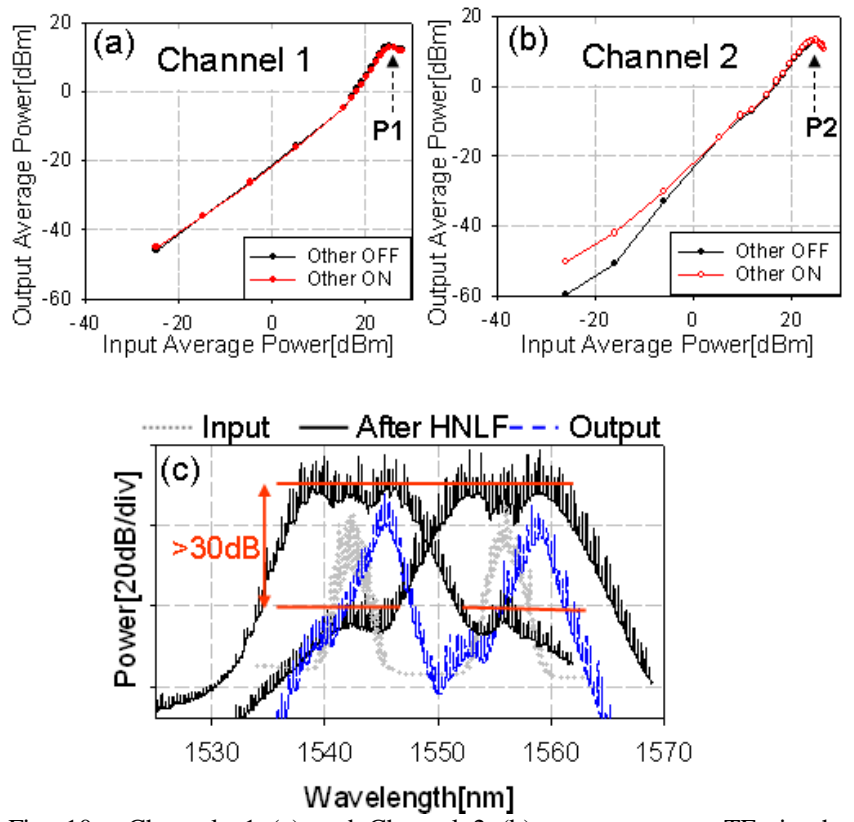

Fig. 10. Channels 1 (a) and Channel 2 (b) average power TFs in the presence (Other ON), or absence (Other OFF), of the second signal. c) Spectra of the channels measured at different points within the regenerator.

along the HNLF. The fiber backscattering mechanism is essentially governed through a Rayleigh scattering process, and originates from sub-wavelength non-uniformities along the fiber, which are found at regions of refractive index variation across the transverse fiber profile. Intuitively, the impact of Rayleigh back-scattering will depend on the regenerator parameters and operational settings, i.e. the spectral position and evolution of the incoming signals and the relative position of the output offset filters. In particular, the strength of this crosstalk is expected to increase as the spectral separation between the two channels decreases. Measurements presented in the following sub-section experimentally verify that this crosstalk does not introduce any significant additional penalty as compared to single-channel operation.

The scheme can be further improved to support 4 channels when a polarization multiplexing configuration is adopted as well, see Fig. 1(c.v) [44]. This approach is illustrated in Fig. 8 (a): the two linearly polarized optical signals are accurately aligned to the two orthogonal birefringence axes of a specially developed polarization maintaining (PM) HNLF used to generate the SPM. This configuration exploits the fast interchannel pulse walk-off due to the large birefringenceinduced differential group delay (DGD) achievable within the PM-HNLF and a further three-fold reduction in XPM due to the tensorial nature of the Kerr effect between orthogonally polarized signals. Fig. 8 (b) shows the numerical simulations of the influence of the fiber birefringence on the transfer function variations clearly showing a cross-talk dependence on the initial time delay, $\tau$, between the two orthogonally copropagating channels [44]. However, as the birefringence is progressively increased, such variations decrease with increasing $\tau$.

\section{B. Experimental validation of the two-channel bi-directional scheme}

Within the TRIUMPH project, we have experimentally demonstrated the simultaneous regeneration of two channels at bit rates exceeding 130Gbit/s using the bi-directional scheme [43] and some of the corresponding results are reported here.

Fig.9 (a) shows the experimental set-up used in this demonstration. Two $40 \mathrm{GHz}$ mode locked fiber lasers operating at 1542nm (Channel 1) and 1556nm (Channel 2) were used to generate $\sim 2 \mathrm{ps}$ pulses. The two signals were amplitude modulated and temporally multiplexed up to 130 Gbit/s. The two corresponding input eye diagrams, measured with an optical sampling oscilloscope with a time resolution of about 800fs, are shown in Fig.9 (b) and Fig.9 (c), respectively. The two channels were then combined together before entering the $2 \mathrm{R}$ regenerator. The corresponding spectrum at the regenerator input is shown in Fig.10 (c) (gray dotted line). In the regenerator the data streams were split with optical filters to allow the signals to be fed into a single HNLF in counterpropagating directions (two HNLF ports). Each optical signal was then separately amplified and fed to the HNLF. Two optical circulators were used to separate the incoming from the outgoing signals at the two fiber input/output ports. The optical parameters of the 310 m-long HNLF, measured at $1550 \mathrm{~nm}$, are as follows: chromatic dispersion of -0.31 $\mathrm{ps} / \mathrm{nm} / \mathrm{km}$, dispersion slope of $0.0031 \mathrm{ps} / \mathrm{nm}^{2} / \mathrm{km}$, nonlinear coefficient of $22 \mathrm{~W}^{-1} \mathrm{~km}^{-1}$ and attenuation of $1.21 \mathrm{~dB} / \mathrm{km}$. The two SPM-broadened spectra of each channel are shown in Fig.10 (c) (black lines), which clearly illustrate the Rayleigh 


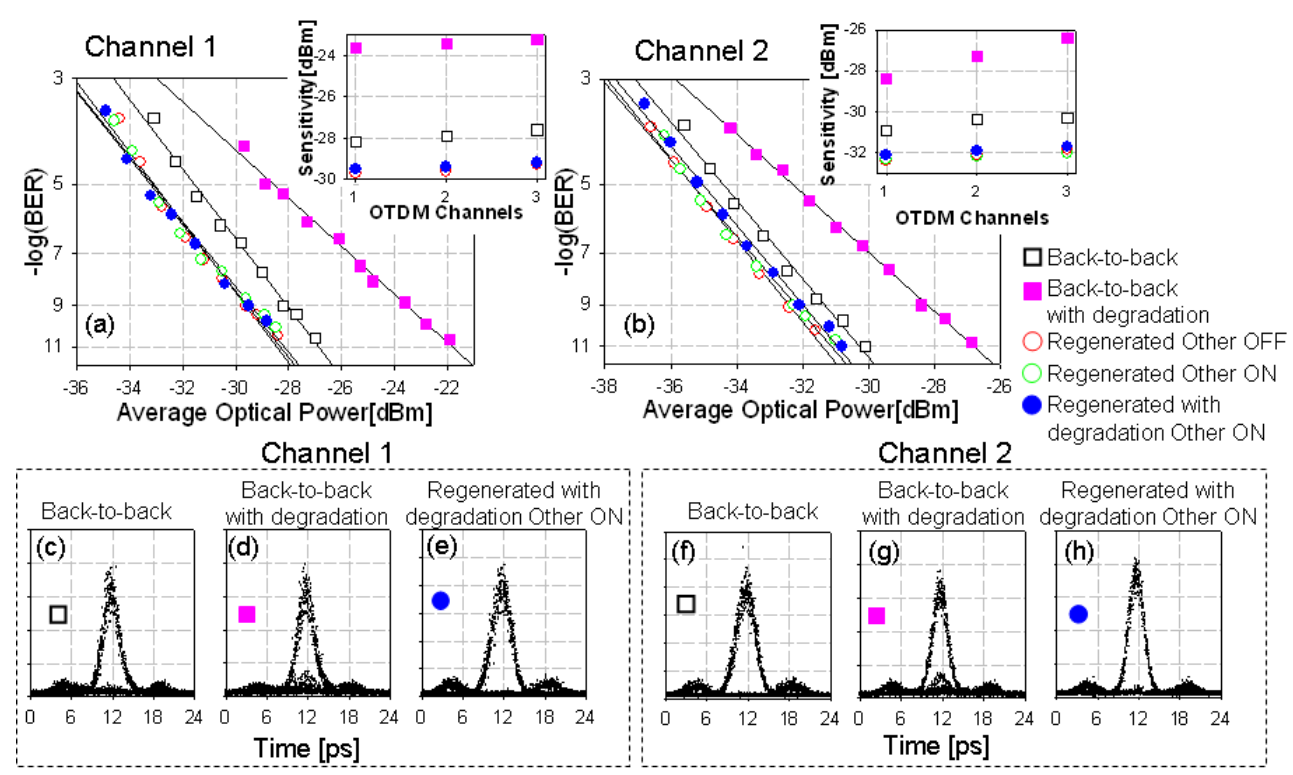

Fig. 11. BER curves for the undistorted (empty symbols) and distorted (filled symbols) back-to-back signals (rectangular), and for the corresponding regenerated (circles) signals for Channel 1 (a) and Channel 2 (b), respectively, in the absence or presence of the interfering channel. Insets: Corresponding receiver sensitivities for the two channels. (c-h) Demultiplexed eye diagrams at the regenerator input/output.

back-scattering (RBS) contribution of the counter-propagating channel [45]. Its main consequence was to reduce the output extinction ratio of the corresponding regenerated channel. However, this back-scattering level was found to be more than $30 \mathrm{~dB}$ below the corresponding transmitted broadened spectrum, causing negligible crosstalk between the two channels as it will be confirmed by the corresponding BER measurements further down. The outgoing signals were then filtered at an offset of $\sim 3 \mathrm{~nm}$ with respect to the incoming carrier wavelengths, in order to preserve the initial channel separation and the corresponding eye diagrams at the regenerator output when both signals were switched on are shown in Fig.9 (d) and Fig.9 (e), respectively. The spectrum at the output of the regenerator, when both signal were multiplexed again for ideally further transmission is shown in Fig. 10 (c), see corresponding blue dashed lines. Finally, the signals were demultiplexed to $40 \mathrm{Gbit} / \mathrm{s}$, using an EAM, see the inset of Fig.9 (a), to allow characterization of the system in terms of BER curves and eye diagrams at $40 \mathrm{Gbit} / \mathrm{s}$.

The power transfer functions of the regenerator for Channel 1 and Channel 2 are plotted in logarithmic scale in Fig.10 (a) and Fig.10 (b), respectively, for the cases that the second channel was switched off (Other OFF) or was operating at the nominal operational input power (Other ON), denoted as P1 and $\mathrm{P} 2$ in the corresponding figures, respectively. The almost identical performance for dual or single channel operation confirms that the regenerator operation is not affected by the presence of a second channel. The small difference at low input powers is attributed to an additional spectral contribution generated by RBS of the counter propagating channel, as discussed already [45]. Furthermore, due to the very low dispersion slope of the HNLF, the two channels (spaced by $\sim 14 \mathrm{~nm}$ ) exhibit quite similar transfer functions.

Bit-error-rate measurements on both channels for both single- and dual-channel operation were then performed (see Fig.11 (a) and Fig.11 (b)). The performance of the regeneration system is very similar for both cases, highlighting the absence of any kind of crosstalk between the two channels. Note that the apparent BER improvement compared to the back-to-back curves is due to a non-optimal transmitter (see eye diagrams of the demultiplexed back to back signals in Fig.11 (c) and Fig.11 (f), respectively), and the wavelength dependence of the EAM used in the demultiplexer, which presents slightly better performance at longer wavelengths. We next artificially degraded the extinction ratio of the input signal to the regenerator, by selecting a sub-optimum bias voltage setting of the lithium-niobate data modulator. The corresponding demultipexed eye diagrams for the two channels are shown in Fig.11 (d) and Fig.11 (g), respectively. After the regenerator, the corresponding BER measurements confirm that complete correction of the $\sim 4 \mathrm{~dB}$ power penalty (at $\mathrm{BER}=10^{-9}$ ) introduced by the signal degradation is achieved at the regenerator output for both channels in the presence of the interfering channel. The corresponding regenerated demultiplexed signals are shown in Fig.11 (e) and Fig.11 (h) when both signals are on.

Questions relating to the extension of the scheme in terms of bit rate (BR) have also been investigated numerically [42]. Systems operating at 160, 320 and $640 \mathrm{Gbit} / \mathrm{s}$ have been considered, for conditions under which the same transfer function reported in the experiment above is maintained, see corresponding design map reported in [51]. The optical parameters of the fiber have also been kept the same, while the fiber length has been allowed to vary according to the BR considered. In particular, to maintain the same regenerative properties, shorter lengths of fiber have to be chosen at higher BRs, so that the ratio between the effective and dispersive lengths remains constant [51]. This, obviously, comes at the 
cost of higher peak power levels at the regenerator input.

The simulation results show that the overall crosstalk level, which is proportional to the power spectral density of the RBS of the second channel across the passband of the offset filter, decreases by about $\sim 3 \mathrm{~dB}$ as the BR is doubled. This implies that there are no evident scalability limitations in terms of BR for the bidirectional scheme.

It is worth emphasizing that the robustness of this multiwavelength regenerating sub-system was confirmed in tests performed on a novel all-optical grooming and regenerating switch for interconnecting $130 \mathrm{Gbit} / \mathrm{s}-\mathrm{OOK}$ core ring and 43 Gbit/s-OOK metro/access ring networks with switching functionality in time, space and wavelength domains [46-47]. This switching node was successfully demonstrated in both laboratory experiments [46] as well as in a real field trial [47].

\section{Experimental validation of the bi-directional and polarization multiplexing scheme}

Within the TRIUMPH project, we have also experimentally demonstrated the simultaneous regeneration of four WDM channels at 10Gbit/s using a bidirectional and polarization multiplexing configuration [44] and some of the corresponding results are reported here.

Fig. 12 shows the experimental set-up of the bi-directional and polarization multiplexing scheme. Four $10-\mathrm{GHz}$ pulsed data streams were generated by gain-switched laser diodes with pulse durations of 7-8 ps. All channels were individually amplitude modulated, amplified and fed into the PM-HNLF via polarization beam combiners (PBCs). Two optical circulators separated the transmitted and the reflected signals from the HNLF at both fiber ends. Finally, a $0.53-\mathrm{nm}$ bandwidth tunable optical filter with a relative offset of about $-0.8 \mathrm{~nm}$ at port 3 of the circulator acted as the reshaping and decision element. The PM-HLNF was $1-\mathrm{km}$ long, has a chromatic dispersion value of $4.2 \mathrm{ps} / \mathrm{nm} / \mathrm{km}$ at $1550 \mathrm{~nm}$ (slow axis), has a nonlinear coefficient of $20 \mathrm{~W}^{-1} \mathrm{~km}^{-1}$ and has a loss of $4.2 \mathrm{~dB} / \mathrm{km}$. Its birefringence value was $7 \times 10^{-4}$ (corresponding to a DGD value of $2.3 \mathrm{ps} / \mathrm{m}$ ) and a nominal polarization extinction ratio of $-30 \mathrm{~dB}$ over $100 \mathrm{~m}$.

Bit-error rate measurements were carried out for the four channels both under single- and multi-channel operation (Fig. 13). No additional power penalty was observed in the two operations, illustrating the absence of strong inter-channel crosstalk amongst the various channels. Finally, by selecting sub-optimum bias voltage setting for the modulator, the channel that presented the worst cross-talk scenario (Channel 3 in our particular case) was artificially degraded. A power penalty improvement of up to $\sim 1.8 \mathrm{~dB}$ (at $\mathrm{BER}=10^{-9}$ ) was achieved under both single and multi channel operation and the good quality of the regenerator was also reflected into the corresponding eye-diagrams, see inset of Fig.13.

Possible extension of this scheme in terms of BR has also been investigated numerically [42], where the scaling towards higher BR is mainly limited by the crosstalk between the copropagating channels. In order to maintain similar performance to the one discussed in the experiment above, and

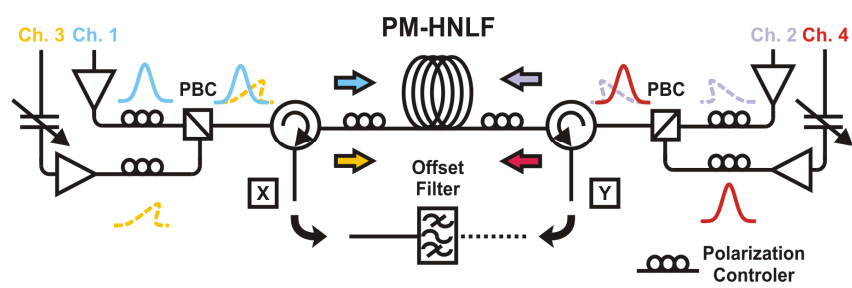

Fig. 12. Experimental set-up of the bi-directional and polarization multiplexing scheme.
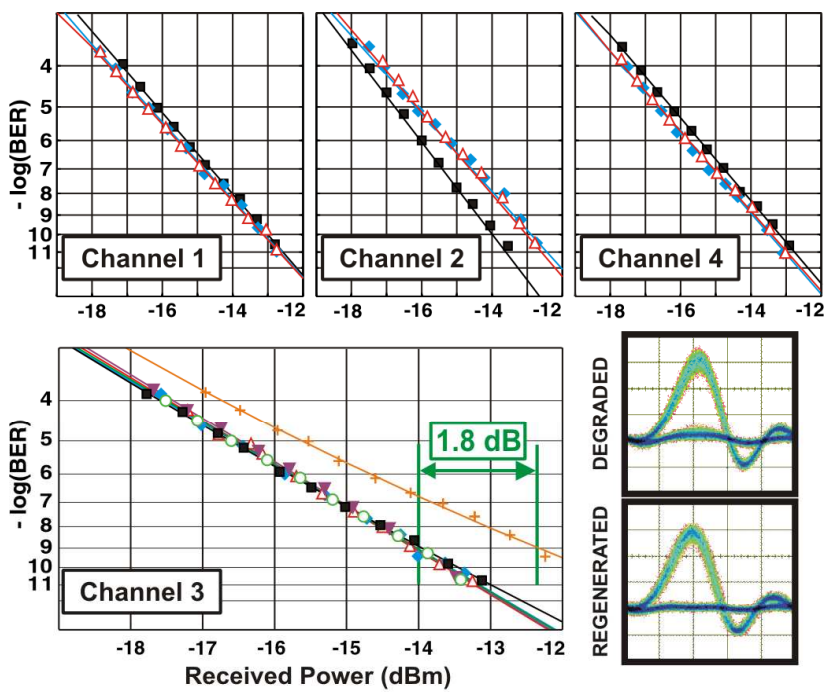

- Back-to-back
$+\begin{aligned} & \text { Degraded input } \\ & \text { signal }\end{aligned}$ Single channel
(w/o degradation)
Single-channel (w. degradation)

$\triangle$ Multi-channel (w/o degradation)

Multi-channel (w. degradation)

Fig. 13. BER measurements of the four channels in the absence and presence of interfering channels. The additional BER curve for Channel 3 corresponds to the degraded signal case. Corresponding eye diagrams of the input degraded and output regenerated data stream.

taking into account that the XPM-induced phase shift is proportional to both the pulse peak power and the DGD, the birefringence value has to scale quadratically with the BR, which could lead to high values of birefringence as we move towards $160 \mathrm{Gbit} / \mathrm{s}$. For example, in case of $33 \% \mathrm{RZ} 40 \mathrm{Gbit} / \mathrm{s}$ signals a minimum DGD value of $1.3 \mathrm{ps} / \mathrm{nm}$ is required to limit the XPM-induced power fluctuations within $+/-10 \%$ at any input power levels along the transfer function.

\section{CONCLUSIONS}

We have reviewed the status of some of the most promising technologies and solutions for multi-wavelength optical regeneration. We have discussed their operational principles and their limits for realistic applications. While in-line optical regeneration clearly represents a key functionality in future long-haul transmission systems, several technical and economical issues remain to be addressed for practical/industrial implementation, the most pressing of which is the mitigation of the inter-channel crosstalk. A variety of approaches and possible solutions were described, which are mainly based on nonlinear interferometer/gates in fibers.

We have then presented the progress towards multi- 
wavelength $2 \mathrm{R}$ all-optical regeneration suitable for high bit rate RZ OOK modulation format signals in optical fibers, as achieved within the European research project TRIUMPH. All of the schemes investigated are based on SPM-induced spectral broadening in HNLFs followed by offset filtering and differ only in their architecture and the resilience they offer to inter-channel nonlinear crosstalk. The uni-directional scheme allows its scalability to a large number of WDM channels that can be used. It also benefits from not requiring any initial demultiplexing of the signals.

The main feature of the bi-directional and polarization multiplexing schemes, on the other hand, is that they offer similar performance and fiber design complexity as their corresponding single-channel devices.

Note that since the beginning of the TRIUMPH project new phase encoded modulation formats, such as (D)PSK, have emerged in long-haul transmission as promising alternatives to the conventional OOK, due to their lower optical signal-tonoise requirements and higher tolerance to nonlinear effects [52-53]. As such, all-optical regenerators for DPSK signals have emerged as an active and important research topic [26, 54-62]. Phase preserving amplitude regenerators for DPSK signals have been investigated in nonlinear amplifying loop mirrors [54-55, 26] or using saturation of FWM interactions caused by pump depletion in nonlinear fibers [56-57]. Both phase and amplitude regeneration can be achieved either by adopting some form of phase-to-amplitude format conversion and then applying amplitude regeneration of the amplitude encoded signal [58], or directly by exploiting the phase squeezing capability and the saturated operating regime of phase sensitive amplifiers (PSAs) in either single- or dualpump configurations using either interferometric configurations based on nonlinear optical loop mirrors or single path fiber-based FWM processes [59-62]. Among these, only Ref. [26] experimentally reports multi-wavelength regeneration using space division multiplexing, while Refs. [63-65] show the potential of in-line multi-wavelength regenerating amplification.

The multi-wavelength regeneration schemes developed within TRIUMPH could be extended to DPSK signals if before the regenerators the WDM signals were phase-toamplitude converted, using for example the approach proposed in [58]. However, this implementation could quickly become complex and costly as the number of WDM channel increases.

\section{ACKNOWLEDGMENT}

The Authors would like to acknowledge the precious contributions of C. Finot, C. Kouloumentas, P. Vorreau and K. Mukasa to the detailed experimental work presented in this paper.

\section{REFERENCES}

[1] H. Hu, E. Palushani, et al., "640 Gbit/s and $1.28 \mathrm{Tbit} / \mathrm{s}$ polarisation insensitive all optical wavelength conversion," Opt. Express 18, 99619966 (2010).
[2] M. Jinno, M. Abe, "All optical regenerator based on nonlinear fibre Sagnac interferometer", Electronics Letters, 28, 14, pp1350, 1992.

[3] J. K. Lucek, K. Smith, "All optical signal regenerator", Optics Letters, 18, 15, pp1226, 1993.

[4] W. A. Pender, P. J. Watkinson, E. J. Greer, A. D. Ellis, "10 Gbit/s all optical regenerator", Electronics Letters, 31, 18, pp 1587, 1995.

[5] W. A. Pender, T. Widdowson, A. D. Ellis, "Error free operation of a 40 Gbit/s all-optical regenerator", Electronics Letters, 32, 6, pp567, 1996.

[6] G. Raybon, et al., "40 Gbit/s pseudo-linear transmission over one million kilometers," Optical Fiber Communication Conference and Exhibit, vol., no., pp. FD10-1- FD10-3, 17-22 Mar 2002.

[7] S. Watanabe, et al., "160 Gbit/s optical 3R regenerator in a fiber transmission experiment," OFC 2003, PD16, 2003.

[8] P.V. Mamyshev, "All-optical data regeneration based on self-phase modulation effect," ECOC, Madrid, Spain, pp. 475, 1998.

[9] A. A. M. Saleh and J. M. Simmons, "Evolution toward the nextgeneration core optical network," J. Lightwav. Technol., vol. 24, no. 9, pp. 3303-3321, Sep. 2006.

[10] J. Leuthold, et al., "40 Gbit/s transmission and cascaded all-optical wavelength conversion over $1000000 \mathrm{~km}$," Electronics Letters , vol.38, no.16, pp. 890- 892, 1 Aug 2002.

[11] T. Akiyama, et al., "Application of Spectral-Hole Burning in the Inhomogeneously Broadened Gain of Self-Assembled Quantum Dots to a Multiwavelength-Channel Nonlinear Optical Device” IEEE Photonics Technology Letters, vol. 12, No. 10, p. 1301-1303, 2000.

[12] M. Sugawara, et al., "Ultrafast optical signal processing based on quantum-dot semiconductor optical amplifiers: Theory and experiment," in Optical Amplifiers and Their Applications, OSA Technical Digest Series (Optical Society of America, 2003), paper WB1.

[13] M. Spyropoulou, S. Sygletos, I. Tomkos, "Study of a multi-wavelength regenerative subsystem based on quantum dot semiconductor optical amplifiers at 40Gbps," OFC/NFOEC, 2007.

[14] A. Shen, et al., "4-channel saturable absorber module for high bit-rate regenerated WDM transmission" Proc. Eur. Conf. Optical Communications, paper 5.4.5, 2002.

[15] Q. T. Le, et al., "WDM compatible $2 \mathrm{R}$ regeneration device based on eight-channel saturable absorber module," Electron Lett. 43 (23), 2007.

[16] M. Nakazawa, E. Yamade. H. Kubota. and K. Suzuki, "10 Gbit/s soliton data transmission over one million kilometers," Electron. Lett., 27. pp. 1270-1272, (1991).

[17] O. Leclerc, et al., "Optical Regeneration at $40 \mathrm{~Gb} / \mathrm{s}$ and Beyond," J. Lightwave Technol. 21, 2779-2790, 2003.

[18] N. Edagawa and M. Suzuki, "Novel all optical limiter using electroabsorption modulators," OFC, ThP1, 2000.

[19] K. Nishimura, R. Inohara, M. Usami, S. Akiba, “ All-optical regeneration by electro-absorption modulator," IEICE Transactions on Electronics, v E88-C, n 3, p 319-326, 2005.

[20] A. Capua, et al., "Cross talk free multi channel processing of $10 \mathrm{Gbit} / \mathrm{s}$ data via four wave mixing in a $1550 \mathrm{~nm}$ InAs/InP quantum dash amplifier," Opt. Express 16, 19072-19077 (2008).

[21] I. Tomkos, et al., "Transparent ring interconnection using multiwavelength processing switches", International Conference on Transparent Optical Networks, paper Mo.B.1.5, 2006.

[22] J. Leuthold, et al., "Optical grooming switch to interconnect access and metro ring networks,": International Conference on Transparent Optical Networks, paper We.C3.4, 207-210, 2008.

[23] O. Leclerc, et al., "Simultaneously regenerated 4 x 40Gbit/s dense WDM transmission over $10,000 \mathrm{~km}$ using single $40 \mathrm{GHz} \mathrm{InP}$ MachZehnder modulator," Electron. Lett. 36, 1574-1575, 2000.

[24] F. Seguineau, et al., "Experimental demonstration of simple NOLMbased $2 \mathrm{R}$ regenerator for $42.66 \mathrm{Gbit} / \mathrm{s}$ WDM long-haul transmissions," in Optical Fiber Communication Conference, 2004 Optical Society of America, Technical Digest on CD-ROM, paper WN4.

[25] S. Boscolo, S. K. Turitsyn, K. Blow, "All-optical passive 2R regeneration for $\mathrm{N}$ x $40 \mathrm{Gbit} / \mathrm{s}$ WDM transmission using NOLM and novel filtering technique," Opt. Commun. 217, 227-232 (2003).

[26] K. Cvecek, et al., "Phase-preserving amplitude regeneration for a WDM RZ-DPSK signal using a nonlinear amplifying loop mirror," Opt. Express 16, 1923-1928, 2008.

[27] M. Asobe, et al., "Noise reduction of $20 \mathrm{Gbit} / \mathrm{s}$ pulse train using spectrally filtered optical solitons," Electronics Letters, Vol. 34 ( 11), 1135 - 1136, 1998. 
[28] E. Desurvire, O. Leclerc, and O. Audouin, "Synchronous in-line regeneration of wavelength-division multiplex solitons signals in optical fibers," Opt. Lett., vol. 21, no. 14, pp. 1026-1028, 1996.

[29] N. Chi, L. Xu, K. S. Berg, T. Tokle, and P. Jeppesen, "All-optical wavelength conversion and multichannel $2 \mathrm{R}$ regeneration based on highly nonlinear dispersion-imbalanced loop mirror," IEEE Photon. Technol. Lett. 14, 1581-1583, 2002.

[30] C. Kouloumentas, et al., "Four-channel all-fibre dispersion-managed 2R regenerator," IEEE Photon. Technol. Lett. 20(13),1169-1171, 2008.

[31] N. S. M. Shah, M. Matsumoto, “ 2R Regeneration of Time-Interleaved Multiwavelength Signals Based on Higher Order Four-Wave Mixing in a Fiber ," IEEE Photon. Technol. Lett. 22 (1), 27-29, 2010.

[32] Y.-K. Huang, I. Glesk, R. Shankar, and P. R. Prucnal, "Simultaneous all-optical 3R regeneration scheme with improved scalability using TOAD," Opt. Express 14, 10339-10344, 2006.

[33] D.V. Kuksenkov, S. Li, M. Sauer, and D. A. Nolan, "Nonlinear Fibre Devices Operating on Multiple WDM Channels," in European Conference on Optical Communications (ECOC'05) 2005, Glasgow, UK.

[34] P. G. Patki, M. Vasilyev, and T. I. Lakoba, "Multichannel All-Optical regeneration," Photonics Society Summer Topical Meeting Series, 172173, 2010.

[35] M. Vasilyev, T. I. Lakoba, and P. G. Patki, "Multi-wavelength AllOptical regeneration," Proc OFC, OWK3 (2008).

[36] I. Tomkos, C. Koulumentas, S. Tsolakidis, "Performance studies of multi-wavelength all-optical $2 \mathrm{R}$ regeneration subsystems based on highly nonlinear fibers," in International Conference on Transparent Optical Networks Rome, Italy, Tu.C1.1, 2007.

[37] T. I. Lakoba, and M. Vasilyev, "A new robust regime for a dispersion managed multichannel 2R regenerator," Opt. Express, 15(16), 1006110074, 2007.

[38] M. Vasilyev and T. I. Lakoba, "All-optical multichannel 2R regeneration in a fibre-based device," Opt. Lett., vol. 30, no. 12, pp 1458-1460, 2005.

[39] C. Kouloumentas, et al., "All-fiberized dispersion-managed multichannel regeneration at $43 \mathrm{~Gb} / \mathrm{s}$," IEEE Photonics Technology Letters, 20(20), 1854-1856, 2008.

[40] T. Ohara, H. Takara, A. Hirano, K. Mori and S. Kawanishi, "40-Gb/s x 4-channel all-optical multichannel limiter utilizing spectrally filtered optical solitons," IEEE Photon.Technol. Lett., 15, pp. 763-765, 2003.

[41] B. Cuenot, and A. D. Ellis, "WDM signal regeneration using a single all optical device”, Opt. Express, vol. 15, pp.11492-11499, 2007.

[42] F. Parmigiani, L. Provost, P. Petropoulos, D. J. Richardson, "Multiwavelength all-optical regeneration techniques," IEEE/LEOS Summer Topicals 2009 Newport Beach 20-22 Jul 2009 (Invited).

[43] F. Parmigiani, et al., "2R regeneration of two $130 \mathrm{Gbit} / \mathrm{s}$ channels within a single fiber", Proc OFC, JThA56, 2009.

[44] L. Provost, et al., "Investigation of Four-Wavelength Regenerator Using Polarization- and Direction-Multiplexing", Photon. Technol. Lett., 20, 1676-1678, 2008.

[45] L. Provost, et al., "Analysis of a two-channel 2R all-optical regenerator based on a counter-propagating configuration," Opt. Express, 16(3), 2264-2275, 2008.

[46] P. Vorreau, et al., "Optical grooming switch with regenerative functionality for transparent interconnection of networks", Optics Express, 17(17), 15173-15185, 2009.

[47] G. Zarris, et al., "Field experiments with a grooming switch for OTDM meshed networking," IEEE Journal of Lightwave Technology, 28(4), 316-327, 2010

[48] K. M. Chong, L. K. Chen, "Optical 3R Regeneration for 10 Synchronous Channels Using Self-Phase Modulation in a Bidirectional Fiber Configuration," OECC'09, WD3 (2009).

[49] L. K. A. Cheng, C. Shu, and M. P. Fok, "All-Optical Multi-Wavelength Extinction Ratio Enhancement via Pump-Modulated Four-Wave Mixing," in National Fiber Optic Engineers Conference, OSA Technical Digest (CD) (Optical Society of America, 2009), paper JThA61.

[50] M. Matsumoto, "Performance Analysis and comparison of optical 3R regenerators utilizing self-phase modulation in fibers," IEEE Journal of Lightwave Technology, 22(6), 1472-1482, 2004.

[51] L. Provost, et al., "Design scaling rules for $2 \mathrm{R}$-optical self-phase modulation-based regenerators," Optics Express, 15,5100-5113, 2007.
[52] A. H. Gnauck, P. J. Winzer, "Optical phase shift-keyed transmission," IEEE J. Lightwave Technol. 23, 115-129, 2005.

[53] C. Xu, X. Liu, and X. Wei, "Differential phase-shift keying for high spectral efficiency optical transmissions," IEEE J. Sel. Topics Quantum Electron. 10 (2), 281-293 (2004).

[54] K. Cvecek, K. Sponsel, G. Onishchukov, B. Schmauss, and G. Leuchs, "2R-Regeneration of an RZ-DPSK signal using a Nonlinear Amplifying Loop Mirror,” IEEE Photon. Technol. Lett., vol. 19, no. 3, pp. 146-148, 2007.

[55] C. Stephan, K. Sponsel, G. Onishchukov, B. Schmauss, and G. Leuchs, "Nonlinear Phase Noise Reduction in a DPSK Transmission System Using Cascaded Nonlinear Amplifying Loop Mirror," IEEE Photon. Technol. Lett., vol. 21, no. 24, pp. 1864-1866, December 2009.

[56] M. Matsumoto, and K. Sanuki, "Performance improvement of DPSK signal transmission by a phase-preserving amplitude limiter," Opt. Express.,vol.15. no. 13, pp.8094-8103, June 2007.

[57] K. Croussore, G. Li, "Amplitude regeneration of RZ-DPSK signals based on four-wave mixing in fibre," Electron. Lett., 43 (3), 177-178, 2007.

[58] M. Matsumoto and H. Sakaguchi, "DPSK signal regeneration using a fiber-based amplitude regenerator," Opt. Express 16, 11169-11175 (2008).

[59] K. Croussore, I. Kim, C. Kim, Y. Han, G. Li, “ Phase-and-amplitude regeneration of differential phase-shift keyed signals using a phasesensitive amplifier," Optics Express 14, 2085-2094, 2006.

[60] K. Croussore and G. Li, "BPSK phase and amplitude regeneration using a traveling-wave phase-sensitive amplifier," IEEE/LEOS Winter Topical Meeting Series, MB1.3, 2008.

[61] K. Croussore and G. Li, "Phase Regeneration of NRZ-DPSK Signals Based on Symmetric-Pump Phase-Sensitive Amplification", IEEE Photon. Tech. Lett. 19 (11) 864-866 (2007).

[62] R.Slavik, et al., "All-optical phase and amplitude regenerator for nextgeneration telecommunications systems," Nature Photonics 4 , 690-695 (2010)

[63] .A. Fragkos, A. Bogris and D. Syvridis, "All-optical Regeneration Based on Phase-Sensitive Non-degenerate Four-Wave Mixing in Optical Fibers," IEEE Photon. Tech. Lett., 2010.

[64] M. Vasilyev, "Distributed phase-sensitive amplification," Optics Express, v 13, n 19, p 7563-7571, 2005.

[65] R. Tang, P. S. Devgan, V. Grigoryan, P. Kumar, M. Vasilyev, "In-line phase-sensitive amplification of multi-channel CW signals based on frequency nondegenerate four-wave-mixing in fiber," Optics Express, v 16, n 12, p 9046-9053, 2008.

Francesca Parmigiani was born in Milan, Italy. She graduated with honours in Electronic Engineering at Politecnico di Milano, Milano, Italy, in 2002, and received the Ph.D. degree in optical communication systems at the Optoelectronics Research Centre (ORC), University of Southampton, Southampton, U.K in 2006.

She is currently a Senior Research Fellow at the ORC. In April 2010 she was awarded a prestigious Postdoctoral Research Fellowships from the Royal Academy of Engineering, in support of her research on the combination of all-optical signal processing and advanced modulation formats. Her research has produced more than 100 papers in journals and conferences in the field of optical communications. Her research interests include ultra-fast all-optical sampling techniques, pulse shaping using specialized fiber Bragg gratings, all-optical nonlinear processing and switches mainly in optical fibers, as well as advanced modulation formats.

Dr Parmigiani is a member of the Optical Society of America (OSA).

Lionel Provost graduated in optics engineering from the Institut National des Télécommunications (Evry, France) in 2000. He received the MSc in Optical and High-Frequency communication systems from the University of Marne-la-Vallée (France). In 2000, he joined Alcatel CIT as a R\&D engineer and was involved in fiber-based components modelling and development. In 2003, he joined Avanex Corp. (France) when he was involved in the design and manufacturing of fibre Bragg Gratings for submarine and terrestrial applications. In 2005, he joined the Optoelectronics Research Centre (Southampton, UK) and since then he was working towards his $\mathrm{PhD}$ on multiwavelength all-optical regeneration. In 2008 he joined Draka Communications and is currently working in fiber optic applications engineering. He has authored or co-authored more than 65 papers in 
technical journals and conferences, and currently holds 18 patent applications or patents.

Periklis Petropoulos was born in Patras, Greece. He graduated from the Department of Electrical Engineering and Information Technology, University of Patras, in 1995, received the M.Sc. degree in communications engineering from the University of Manchester Institute of Science and Technology, Manchester, U.K., in 1996, and the Ph.D. degree in optical telecommunications from the Optoelectronics Research Centre (ORC), University of Southampton, Southampton, U.K. in 2000. He is currently a Reader at the ORC.

His research interests include all-optical processing and switching in optical fibers; pulse manipulation for optical communications using fiber Bragg gratings, including applications in optical correlation systems for the implementation of optical code division multiple access and optical packet switched systems; silica and compound glass holey fibers and their nonlinear applications; and fiber lasers. His research has produced more than 240 papers in journals and conferences in the field of optical physics and optical communications. Dr Petropoulos is a member of the Optical Society of America.

David J. Richardson was born in Southampton, U.K., in 1964. He received the B.Sc. degree and the Ph.D. degree in fundamental physics from Sussex University, U.K., in 1985 and 1989, respectively. In May 1989, he was a Research Fellow at the then recently formed Optoelectronics Research Centre (ORC), Southampton University, U.K. He is currently a Deputy Director at the ORC, responsible for much of the ORC's fiber-related activities. He is one of the founders of Southampton Photonics Incorporated, a university spin-off venture that has successfully commercialized elements of high-power laser technology developed within the ORC.

His current research interests include amongst others: microstructured fibers, high-power fiber lasers, short pulse lasers, optical fiber communications, and nonlinear fiber optics. Prof Richardson has published more than 700 conference and journal papers in his time at the ORC, and produced over 20 patents. He is a frequent invited speaker at the leading international optics conferences in the optical communications, laser and nonlinear optics fields and is an active member of both the national and international optics communities. He was awarded a Royal Society University Fellowship in 1991 in recognition of his pioneering work on short-pulsed fiber lasers, and was made a Fellow of the Optical Society of America in 2005. He was elected as a Fellow of the Royal Academy of Engineering in recognition for his contributions to engineering in 2009.

Wolfgang Freude received the Dipl.Ing. (M.S.E.E.) and the Dr.Ing. (Ph.D.E.E.) degrees in Electrical Engineering in 1969 and 1975 from the University of Karlsruhe, respectively. He is Professor at the Institute of Photonics and Quantum Electronics, Karlsruhe Institute of Technology (KIT). His research activities are in the area of high-density integrated-optics with a focus on silicon photonics, photonic crystals and semiconductor optical amplifiers, and in the field of low-energy opto-electronic devices and protocols for optical access networks. He has published more than 190 papers, coauthored a book entitled "Optical Communications" (Springer 1991, in German), and authored and coauthored two book chapters on "Multimode Fibres" (Handbook of Optical Communications, Springer 2002, in German) and "Microwave Modelling of Photonic Crystals" (Photonic Crystals, Wiley-VCH 2004). Prof. Freude is an Honorary Doctor of the Kharkov National University of Radioelectronics, Kharkov, Ukraine, and a member of VDE/ITG, IEEE, and OSA.

Juerg Leuthold was born in 1966 in Switzerland. He has a Ph.D. degree in physics from the Swiss Federal Institute of Technology (ETH) Zürich for work in the field of integrated optics and all-optical communications.

From 1999 to 2004 he has been affiliated with Bell Labs, Lucent Technologies in Holmdel, USA, where he has been performing device and system research with III/V semiconductor and silicon optical bench materials for applications in high-speed telecommunications. Since July 2004 he is a full Professor at Karlsruhe Institute of Technology (KIT), where he is heading the Institute of Photonics and Quantum Electronics.

Juerg Leuthold is a fellow of the Optical Society of America, a member of the Heidelberg Academy of Science and has been and is serving the community in many technical program committees such as OFC, ECOC,
CLEO and many others. Besides these activities in committees he is currently chairing the Photonics Division of the Optical Society of America.

Andrew D. Ellis received the B.Sc. degree in physics with a minor in mathematics from the University of Sussex, Brighton, U.K., in 1987. He received the $\mathrm{Ph} . \mathrm{D}$. degree in electronic and electrical engineering from The University of Aston in Birmingham, Birmingham, U.K., in 1997 for his study on all optical networking beyond $10 \mathrm{Gbit} / \mathrm{s}$.

He previously worked for British Telecom Research Laboratories as a Senior Research Engineer investigating the use of optical amplifiers and advanced modulation formats in optical networks and the Corning Research Centre as a Senior Research Fellow where he led activities in optical component characterisation. Currently, he heads the Transmission and Sensors Group at the Tyndall National Institute in Cork, Ireland, where he is also a member of the Department of Physics, University College Cork. He research interests include the evolution of core and metro networks, and the application of photonics to sensing. He has published over 100 journal papers and over 20 patents in the field of photonics.

Dr. Ellis is a member of the Institute of Physics and the Institute of Engineering Technology, and is a Chartered Physicist. He acts as a reviewer for IEEE Journal of Lightwave Technology and IEEE Photonics Technology Letters and as an associated editor for Optics Express. He has served as member of the Technical Program Committees for many international conferences, including OFC/NFOEC and ECOC etc.

Ioannis Tomkos is with the Athens Information Technology Center (AIT) since Sep 2002 and an Adjunct Faculty at the Information Networking Institute of Carnegie-Mellon University, USA (2002-2010). In the past, he was senior scientist (1999 - 2002) at Corning Inc. USA and research fellow (1995 - 1999) at University of Athens, Greece. At AIT, he served as Associate Dean (2004-2009) and he founded and serves as the Head of the "High Speed Networks and Optical Communication (NOC)" Research Group that was/is involved in many industry and EU funded research projects (including 7 running FP7 EU projects) within which Dr. Tomkos is representing AIT as Principal Investigator and has a consortium-wide leading role (project leader/technical manager/workgroup leader).

Dr. Tomkos has received (2006) the prestigious title of "Distinguished Lecturer" of IEEE Communications Society. Together with his colleagues and students he has authored over 350 scientific articles and presentations (over 180 IEEE sponsored items), including about 100 Journals/Magazines/Book chapters. Dr. Tomkos has served as the Chair of the International Optical Networking Technical Committee of IEEE Communications Society (20072008) and the Chairman of the IFIP working group on "Photonic Networking" (2008-2009). He is currently the Chairman of the OSA Technical Group on Optical Communications (2009-2010) and the Chairman of the IEEE Photonics Society Greek Chapter (2010). He has been General Chair, Technical Program Chair, Subcommittee Chair, Symposium Chair or/and member of the steering/organizing committees for the major conferences (e.g. OFC, ECOC, IEEE GlobeCom, IEEE ICC, ONDM, BroadNets, etc.) in the area of telecommunications/networking (more than 100 conferences/workshops). In addition he is a member of the Editorial Boards of the IEEE/OSA Journal of Lightwave Technology, the IEEE/OSA Journal of Optical Communications and Networking, the IET Journal on Optoelectronics, and the International Journal on Telecommunications Management. He is a Fellow of the IET. 